\title{
Clinical impact of EUS treatment of walled-off pancreatic necrosis with dedicated devices
}

\section{(ㄷ)(우)(웅}

\author{
Authors \\ Institutions \\ 1 Endoscopy Service, Department of Diagnostic and \\ Therapeutic Services, IRCCS-ISMETT (Istituto \\ Mediterraneo per i Trapianti e Terapie ad alta \\ specializzazione), Palermo, Italy \\ 2 Research Office, IRCCS-ISMETT (Istituto Mediterraneo \\ per i Trapianti e Terapie ad alta specializzazione)
}

Ilaria Tarantino ${ }^{1}$, Dario Ligresti ${ }^{1}$, Fabio Tuzzolino ${ }^{2}$, Luca Barresi ${ }^{1}$, Gabriele Curcio ${ }^{1}$, Antonino Granata ${ }^{1}$, Mario Traina ${ }^{1}$

events (AEs) and death. As an alternative, endoscopic ultrasound-guided cysto-gastro-anastomosis has emerged as an effective treatment for WOPNs. Recently a new dedicatedlumen apposing metal stent (LAMS) has been used with satisfactory results in treating peri-pancreatic fluid collections, including WOPNs. The primary outcomes of this study were to evaluate survival and clinical success. Sec- submitted 15.12.2016

accepted after revision 29.3.2017

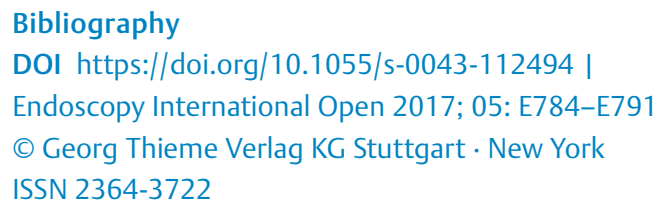

Corresponding author

Ilaria Tarantino, MD, Endoscopy Service, Department of Diagnostic and Therapeutic Services, IRCCS-ISMETT, Via Tricomi 5, 90127 Palermo, Italy

Fax: +390912192400

itarantino@ismett.edu

\section{ABSTRACT}

Background and study aims Walled-off pancreatic necrosis (WOPN) represents the major risk factor for sepsisrelated multiple organ failure. Surgical debridement is an invasive approach associated with high rates of adverse ondary outcomes included: technical success, adverse events and recurrence rate.

Patients and methods All consecutive patients with infected WOPN between February 2014 and June 2016 were retrospectively reviewed. Patients underwent placement of a new LAMS incorporated in an electrocautery-enhanced delivery system and direct endoscopic necrosectomy (DEN). DEN was performed immediately after stent deployment and repeated every 3 to 7 days until complete resolution.

Results In the study period we treated 20 consecutive patients with infected WOPN using the new LAMS. Technical success was achieved in $95 \%$ of patients. Clinical success was achieved in $73 \%$ and $84.2 \%$ of patients at 1 and 3 months, respectively. Survival rate was $84.2 \%$ and $79 \%$. Mean length of hospital stay was 19 days (range $3-43$ ). No AEs occurred. Patients were followed up after stent retrieval for a mean time of 554,7 days (range $70-986$ ) and no recurrence was observed.

Conclusions DEN following "1-step, exchange-free" LAMS positioning recorded excellent results. We believe that simplicity of procedure plays a key role in terms of safety.

\section{Introduction}

According to the Atlanta classification [1], walled-off pancreatic necrosis (WOPN) is an encapsulated collection of fluid and solid debris that usually develops more than 4 weeks after an episode of acute necrotizing pancreatitis. Infected pancreatic necrosis, which is a major risk factor for sepsis-related multiple organ failure, is the main life-threatening complication of acute pancreatitis. Historically, symptomatic infected WOPN was managed by surgical debridement, associated with high rates of adverse events (AEs) (34 to 95\%), death (11 to 39\%) and long-term pancreatic insufficiency [2-7]. As an alternative to open necrosectomy, less invasive techniques, including percutaneous drainage was used to drain WOPN [8]. Imaging-guided percutaneous drainage techniques require an indwelling catheter and repeat interventions for catheter upsizing. In addition, percutaneous drainage is associated with AEs such as catheter occlusion, secondary infections, and fistula formation [9]. Endoscopic ultrasound (EUS)-guided cystogastroduodenostomy with plastic stent placement has emerged as an effective nonsurgical treatment for WOPN. Although outcomes of transluminal drainage are comparable to those with surgery in management of pseudocysts, success rates are lower for manage- 
ment of WOPN because of the presence of necrotic solid debris [10].

Direct endoscopic necrosectomy (DEN) is a minimally invasive technique for performing debridement of the cyst cavity with success rates of $75 \%$ to $91 \%$. Bakker et al. demonstrated in a randomized trial [11] the superiority of transgastric endoscopic necrosectomy over surgical necrosectomy. However, the median number of required procedures ranges from 3 to 5 , in addition to lengthy hospital stays (average stay 12-21 days) [12-15]. To overcome the need for repeat interventions and extended hospital stays, fully covered self-expandable metal stents (FCSEMS) were introduced for treating patients with WOPN. However even if FCSEMSs are larger in diameter they can migrate [16]. In initial experience, FCSEMSs designed for other indications were used [17-21]. AEs, such as stent migration and bleeding, have been described, highlighting the need for dedicated FCSEMSs for this procedure.

Recently, a new dedicated-lumen apposing FCSEMS called "Axios stent" (Boston Scientific, Natick Mass, US) has been used for EUS drainage of pancreatic fluid collections (PFC), including WOPN [22-24]. This large-diameter biflanged lumen apposing metal stent (LAMS) is specifically designed to create an anastomosis between the cyst cavity and the gut lumen. The stent is deployed over a guidewire introduced inside the collection by a standard 19G FNA needle (needle-based technique). In our study, however, we used a new device in which the LAMS is contained in an electrocautery-enhanced delivery system avoiding multiple accessory exchanges to accomplish the drainage. In a recent large multicenter study, EUS-guided drainage with this new device was found to be safe, easy to perform, and a highly effective minimally invasive treatment modality for treating all symptomatic PFCs including WOPN [25]. The aim of this study was to evaluate the clinical efficacy of EUS-guided drainage of WOPN with this dedicated device and subsequent DEN.

\section{Patients and methods}

All consecutive patients with infected WOPN who underwent LAMS stent placement and DEN between February, 2014 and June, 2016 were reviewed. All patients gave their informed consent. WOPN was defined accordingly to the revised 2012 Atlan-

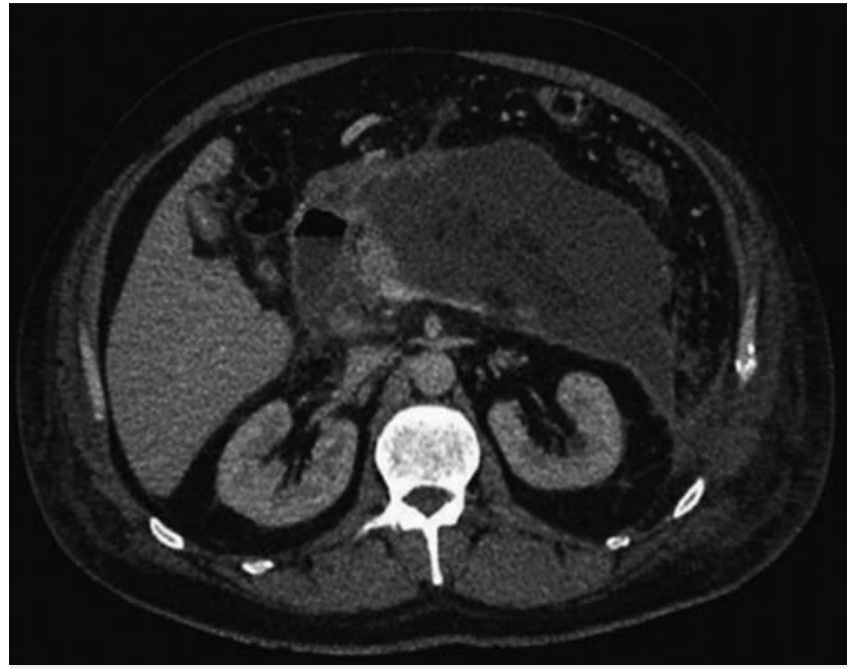

- Fig. 1 CT view of a WOPN: irregular thick-wall fluid collection involving the pancreatic and peripancreatic space.

ta classification [26] and characterized by magnetic resonance imaging (MRI) or computed tomography (CT) ( Fig. 1).

The protocol for retrospective review of the cases was approved our institute's Ethics Committee.

\section{Stent device}

The device (Axios-EC (electrocautery enhanced); Boston Scientific) is a through-the-scope LAMS delivery system with an electrocautery wire at the distal tip ( $\triangleright$ Fig.2a). The stent is composed of braided nitinol that is fully covered with silicone. It has bilateral double-walled anchoring flanges ( $\mathbf{F i g . 2 b}$, - Fig. 2c) designed to hold the stomach or duodenal wall in direct apposition to WOPN wall. The stent is delivered through a $9 \mathrm{~F}$ or $10.8 \mathrm{~F}$ catheter which is Luer-locked to the inlet port of the endoscope instrumentation channel to provide controlled deployment of the stent by the echoendoscopist. The electrocautery tip allows direct passage of the catheter into the collection.

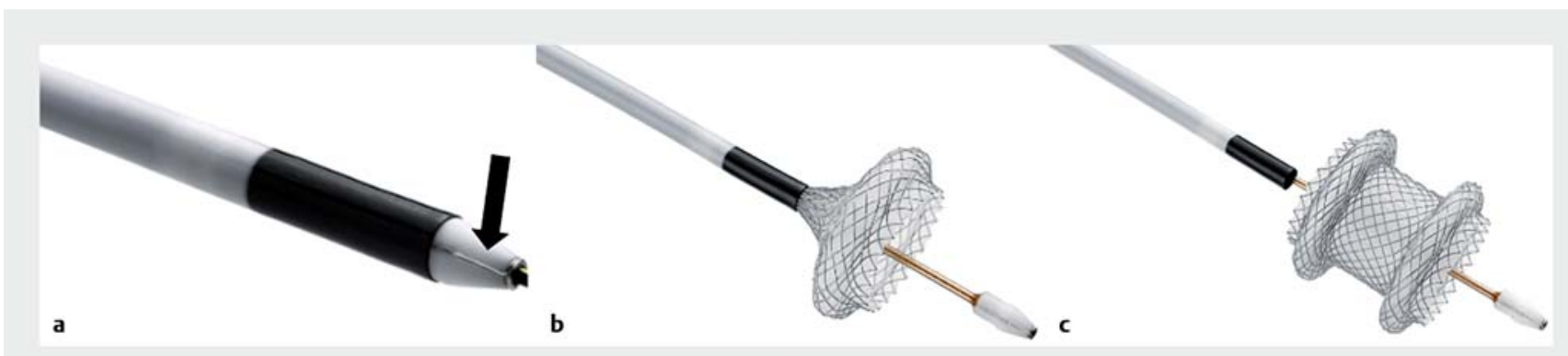

Fig. 2 a Electrocautery wire (arrow) at the distal tip of the LAMS delivery system. b Partially released stent (distal flange). c Fully released stent. 

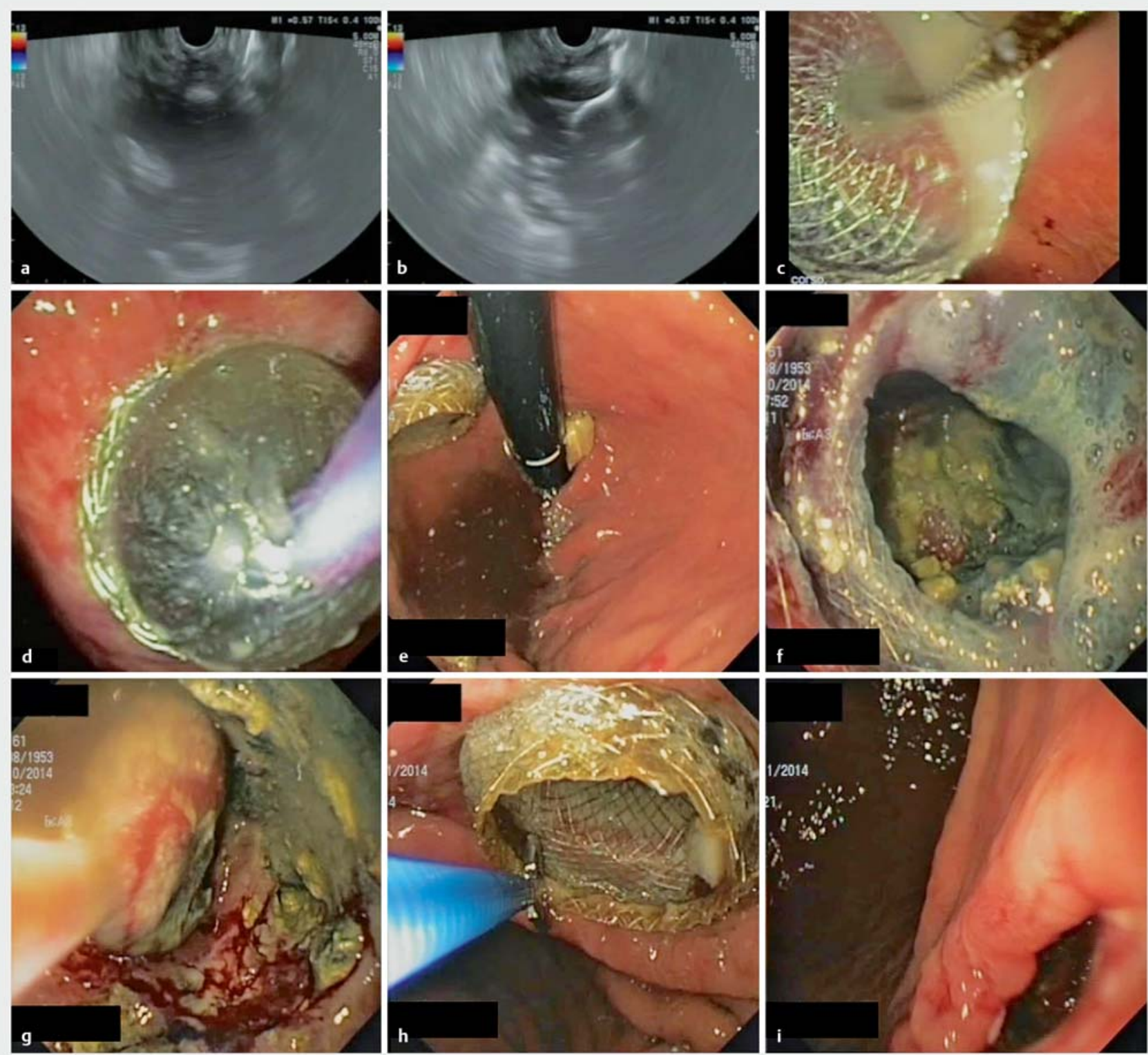

- Fig. 3 EUS-guided drainage with LAMS and necrosectomy in a patient with an infected WOPN. a EUS view of the WOPN, and $\mathbf{b}$ of the distal flange of the stent opened inside the collection. $\mathbf{c}$ Endoscopic view of the proximal flange of the stent. $\mathbf{d}$ Balloon dilation of the stent. e Endoscopic view of the stent correctly positioned with the proximal flange in the gastric cavity. $\mathbf{f}$ View through the stent of necrotic tissue. $\mathbf{g}$ Endoscopic view from inside the WOPN: retrieval of a fragment of necrosis with a standard net. h Stent removal with a biopsy forceps. i Cystogastrostomy immediately following stent removal.

\section{Procedure}

All procedures were performed under general anesthesia. Procedures specifically planned for LAMS retrieval (in the case of pre-procedural imaging study confirmed the resolution of WOPN) were performed under deep sedation. A therapeutic linear echoendoscope (GF-UCT180, Olympus, Tokyo, Japan) was used in all cases. Pre-procedural broad-spectrum antibiotics were administered systemically. The collection was directly accessed using the LAMS which was advanced into the cavity under EUS guidance using the electrocautery tip. Since the stent is very well visible sonographically, the distal flange was then deployed under EUS control, whereas the proximal flange was deployed under endoscopic view without need of fluoroscopic assistance ( $\mathbf{F i g . 3}$ ). After LAMS deployment ( $\mathbf{F i g . 4 )}$ ), a standard gastroscope was inserted to perform immediate DEN. The lumen of the stent was dilated up to its maximum diameter using balloon dilators (CRE Balloon, Boston Scientific). As much of the visualized devitalized tissue that could be removed was removed and $\mathrm{H}_{2} \mathrm{O}_{2}$ irrigation was done at each session. A variable amount, ranging from 100 to 500 cc of $3 \%$ hydrogen peroxide at 1:3-1:2, was sprayed under direct vision on the adherent necrotic tissue, followed by irrigation with saline 


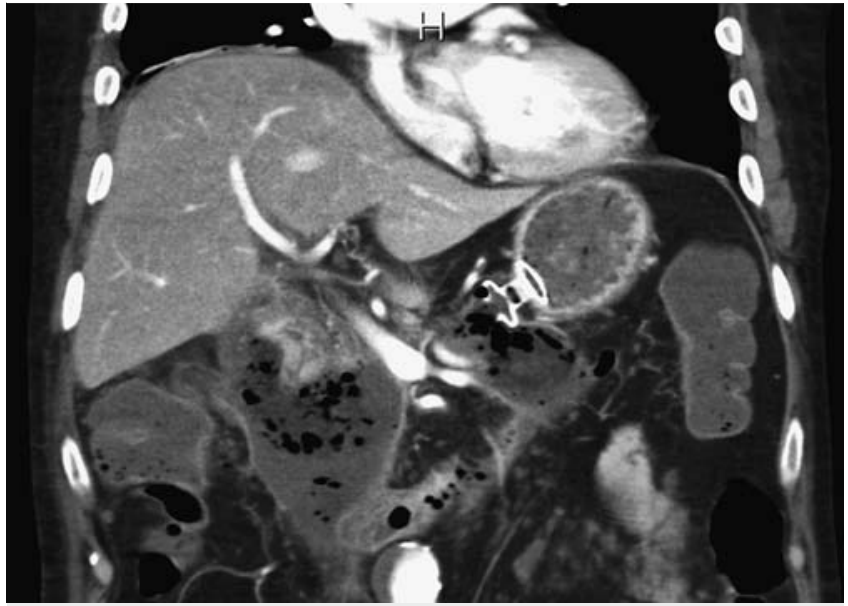

- Fig.4 CT longitudinal reconstruction of a cystogastrostomy with LAMS.

and suction. Repeat DEN was performed through the stent usually every 3 to 7 days in an inpatient setting until complete resolution as confirmed endoscopically. The resolution was further confirmed by cross-sectional imaging (CT/MRI) after LAMS removal. Endoscopic extraction nets, baskets, snares, and hydrogen peroxide irrigation were used during endoscopic debridement with the goal of visualizing pink granulation tissue lining the wall of collection. In some cases, a mechanical lithotriptor was used for fragmentation of large necrotic fragments. All procedures were performed using carbon dioxide inflation.

Post-procedural intravenous antibiotics were given, adjusted according to results of culture tests or stopped if clinical conditions improved. According to clinical conditions patients were given an oral diet 24 hours after the first procedure. For patients unable to do that, an enteral feeding tube was placed.

Stent removal was undertaken with a biopsy forceps. Patients underwent a CT/MRI 1 month after LAMS removal and were then followed at 3-months intervals in an outpatient setting. Further imaging examinations were performed if clinically needed during the follow-up period.

\section{Outcome parameters}

The primary outcomes of this study were survival and clinical success. Survival was evaluated at 1 and 3 months from the first procedure. Clinical success was defined as recovery from sepsis and other WOPN-related symptoms associated with disappearance of WOPN without the need for additional percutaneous or surgical intervention.

Secondary outcomes included technical success, defined as satisfactory access and drainage following correct placement of the LAMS; AEs included bleeding, perforation, spontaneous stent migration defined as interprocedural dislodgement of the stent with worsening of clinical conditions, or other events requiring any interventional treatment; and recurrence rate defined as clinical or radiological relapse at 3 months. Number of endoscopic procedures, procedure time, duration of hospital stay and stent indwelling time were further recorded.
- Table 1 Characteristics of 20 patients with WOPN who underwent EUS-guided drainage with LAMS.

\section{Characteristics}

Patients, $n$ 20

Age, mean (SD) $58.7( \pm 16)$

Male:female 14:6

Etiology of pancreatitis, $n(\%)$

- Biliary $13(65)$

- Idiopatic 3 (15)

- Cystic fibrosis 1 (5)

- Pancreas divisum 1 (5)

- Postoperative fistula

- Chronic pancreatitis

WOPN diameter $(\mathrm{cm})$, mean (range)

$12.5(5-20)$

EUS, endoscopic ultrasound; LAMS, lumen-apposing metal stent; WOPN, walled-off pancreatic necrosis

\section{Data analysis}

To assess the primary objective of the study, survival at 1 and 3 months was evaluated with the proportion and interval confidence ( $\mathrm{Cl} 95 \%$ ). Survival data were summarized with the Kaplan-Meier estimator. Clinical and technical success and the secondary endpoint "adverse events" were analyzed with the same method.

To assess other secondary endpoints (number of endoscopic procedures and procedure time) mean and standard deviation (SD) with interval confidence ( $\mathrm{Cl} 95 \%$ ) were estimated. To assess duration of hospital stay and indwelling time, median and interquartile range (IQR) were estimated.

All statistical analysis was performed with SAS 9.4 (SAS Institute Inc., Cary, NC, US).

\section{Results}

\section{Patient demographics and WOPN characteristics}

Between February 2014 and June 2016 we treated 20 patients with infected WOPN. Patient and WOPN characteristics are summarized in $>$ Table 1.

\section{Procedure}

Successful insertion of a LAMS into the WOPN (technical success) was achieved in 19 (95\%) patients. Unsuccessful placement of LAMS in 1 patient was a result of stent maldeployment due to abundant solid component of the necrotic tissue.

Fourteen (14) patients (73.7\%) had transgastric drainage, 1 patient $(5.3 \%)$ transduodenal drainage and 4 patients $(21 \%)$ multiple drainage ( 2 transgastric and transduodenal; 2 transgastric and transgastric).

A $15-\mathrm{mm}$ internal diameter and $10-\mathrm{mm}$ long stent was used in 17 patients, a $10 \mathrm{~mm} \times 10 \mathrm{~mm}$ stent in 2 patients with medi- 
- Table 2 Results and technical characteristics of procedures.

\begin{tabular}{|c|c|}
\hline \multicolumn{2}{|l|}{ Results and characteristics } \\
\hline Technical success, n (\%) & $19 / 20(95)$ \\
\hline Clinical success at 1 month, $\mathrm{n}(\%)$ & $15 / 19(79)$ \\
\hline Clinical success at 3 months, $\mathrm{n}(\%)$ & $16 / 19(84,2)$ \\
\hline Survival rate at 3 months, $\mathrm{n}(\%)$ & $16 / 19(84,2)$ \\
\hline Adverse events, $\mathrm{n}$ & 0 \\
\hline \multicolumn{2}{|l|}{ Site of drainage, $\mathrm{n}(\%)$} \\
\hline - Transgastric & $14(73,7)$ \\
\hline - Transduodenal & $1(5,3)$ \\
\hline Multiple drainage, n (\%) & $4(21)$ \\
\hline - Transgastric + transduodenal & 2 \\
\hline - Transgastric + transgastric & 2 \\
\hline \multicolumn{2}{|l|}{ Stent dimension (diameter $\times$ lenght) } \\
\hline - $15 \mathrm{~mm} \times 10 \mathrm{~mm}$ & 12 \\
\hline - $10 \mathrm{~mm} \times 10 \mathrm{~mm}$ & 2 \\
\hline - $15 \mathrm{~mm} \times 10 \mathrm{~mm}+15 \mathrm{~mm} \times 10 \mathrm{~mm}$ & 1 \\
\hline - $15 \mathrm{~mm} \times 10 \mathrm{~mm}+8 \mathrm{~mm} \times 8 \mathrm{~mm}$ & 4 \\
\hline Necrosectomy sessions, mean (range) & $3.9(1-9)$ \\
\hline Single procedure duration (min), mean (SD) & $39( \pm 18)$ \\
\hline Duration of hospital stay (days), median (IQR) & $15(20,5)$ \\
\hline Indwelling time of LAMS (days), median (IQR) & $14(21,5)$ \\
\hline $\begin{array}{l}\text { Indwelling time of the second LAMS (days), } \\
\text { median (IQR) }\end{array}$ & $37(43)$ \\
\hline Follow up (days), mean (range) & $554.7(70-986)$ \\
\hline LAMS, lumen-apposing metal stent & \\
\hline
\end{tabular}

um-size infected lesions. An $8 \mathrm{~mm} \times 8 \mathrm{~mm}$ stent was used in 4 patients who needed multiple drainage with transduodenal access. Once the LAMS was positioned, balloon dilation was performed in all cases to allow for immediate DEN. A mean of 3.9 (range 1 -9) sessions of DEN per patient were necessary to obtain complete clearance of the WOPN. Mean procedure duration was 39 minutes (SD \pm 18 ). Procedural characteristics are summarized in $>$ Table 2.

\section{Outcomes}

Survival rate was $84.2 \%$ and $79 \%$ at 1 and 3 months, respectively, after procedure ( $\mathbf{F i g . 5}$ ). Two patients died from WOPNrelated sepsis: the first underwent percutaneous drainage as first treatment in another hospital and was admitted to our institution in severe clinical condition; the patient died from MOF 4 days after the second session of DEN. The second patient was transferred to our institution from another hospital in septic shock. This other patient died from MOF on day 12 of intensive care unit stay and 3 DEN sessions. In both cases no stent obstruction was observed intraprocedurally or at control imaging

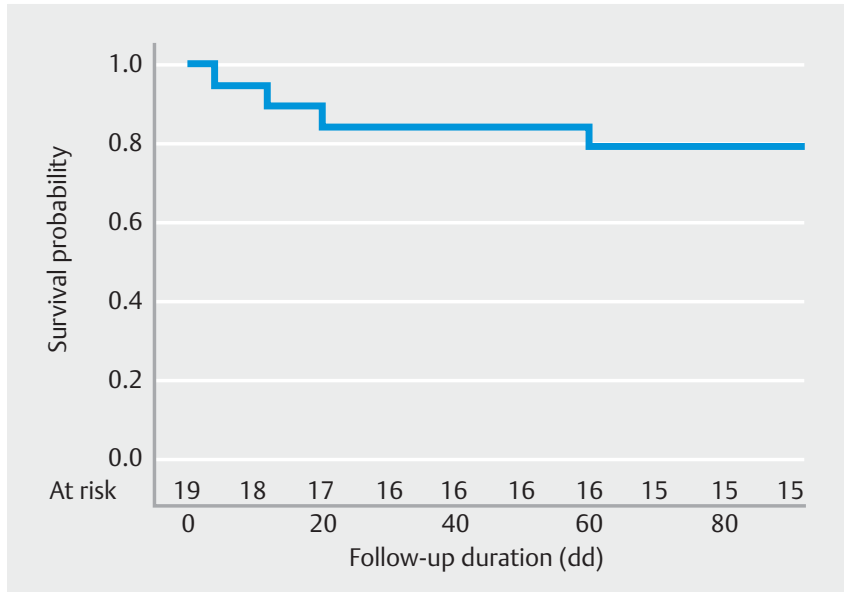

- Fig. 5 Kaplan-Meier plot estimating survival of patients with WOPN treated with EUS-guided LAMS placement, drainage, and necrosectomy.

studies. Two patients died of other causes: 1 of acute respiratory distress syndrome due to H1N1 infection; the other reached a 2-month WOPN-related clinical success, but died of liver failure and cystic fibrosis in the follow-up period.

Clinical success was then achieved in $79 \%$ and $84.2 \%$ of patients at 1 and 3 months, respectively, after procedure. Median duration of hospital stay was 15 days (IQR 20.5). The LAMS was removed after 14 days (median) (IQR 21.5). For patients who underwent multiple drainage, a second LAMS was removed after 37 days (median) (IQR 43). There were no AEs in the follow-up period. Patients were followed up after stent retrieval for a mean time of 554.7 days (range $70-986$ ). No recurrence was observed. Outcomes are summarized in $>$ Table 2.

\section{Discussion}

We retrospectively evaluated a cohort of patients with infected WOPN who underwent EUS-guided drainage of collection using an electrocautery-enhanced LAMS. We found that stent placement was easy and technically successful in all but 1 patient, with very high technical and clinical success rates of $95 \%$ and $84.2 \%$, respectively. Furthermore, no AEs occurred in the follow-up period.

Although there have been multiple small case series and case reports, and a few studies demonstrating the efficacy of LAMS for treatment of PFCs (including WOPN) [22, 25, 27, 28], to our knowledge this is the first study on experience in using LAMS with electrocautery-enhanced delivery system exclusively in WOPNs.

In a retrospective multicenter study [29] conducted on 47 patients using a LAMS positioned with the standard needle-based technique (NAGI stent, TaeWoong Medical Co, Ltd, Gyeonggido, South Korea), the initial clinical success rate was only $76.6 \%$ even though more than $50 \%$ of the PFCs were sterile pseudocysts. Moreover, high rates of early (18.6\%) and late (26\%) AEs, which included stent migration, infection because of stent occlusion, and tissue ingrowth were observed, causing the au- 
thors to conclude that FCSEMSs have significant limitations for management of PFCs.

Conversely, in a multicenter prospective European study [30] conducted in 15 tertiary care centers, on 61 patients (75\% with a WOPN), using the same LAMS we used in this study (but with a needle-based positioning technique), the results were much more encouraging. Clinical success was achieved in $93 \%$ of patients with PFCs and in $81 \%$ of patients with WOPN. Stent migration or stent dislodgement during necrosectomy, and major AEs, including infection and perforation, were observed in 10 patients (18\%). This difference in reported clinical effectiveness between the 2 studies may be related to the design of the stent used.

The superiority of LAMS over plastic stents has been demonstrated by Mukai S. et al [31]. in terms of reduction of procedure time and of number of procedures per patient. This is because the large diameter of the stent allows easy access through the stent into the collection and repeated sessions can be performed. However, in this study different kinds of LAMS, with heterogeneous characteristics, were compared. Thus, in every published study on WOPN drainage and necrosectomy using LAMS, a needle-based positioning technique has been used, affecting outcomes in terms of complexity of procedure. Rinninella et al. [25] in their multicenter study reported successful electrocautery-enhanced LAMS EUS-guided drainage in a heterogeneous cohort of patient with PFCs. This study included WOPN for only $55.9 \%$.

In our study, the drainage procedure was technically successful in all but 1 patient. Direct penetration of the WOPN without the need to puncture with needle and place a guidewire was performed in all patients. This 1-step exchange-free approach should be preferred because it makes the procedure easier and faster to perform than when a needle is used.

LAMS dislodgement has been reported in 1 patient in whom the cystogastrostomy tract had been dilated up to $15 \mathrm{~mm}$ before placement of a 10-mm stent [22]. In another study, both spontaneous migration, with no clinical consequences for the patient, and stent dislodgement were reported [32]. Walter et al. reported 3 stent migrations and 3 dislodgments during necrosectomy. Also in the study by Chandran et al. evaluating NA$\mathrm{GI}^{\mathrm{TM}}$ stent, a high migration rate was reported: 4 cases $(7.4 \%)$ during DEN, 1 case $(1.9 \%)$ of stent migration into the fistula tract, and $6(11.1 \%)$ spontaneous stent migrations.

However, it is important to consider that these reported cases of migration and dislodgment include both WOPN and pseudocysts. Furthermore, these cases involved the needlebased positioning technique, which could have affected the outcome due to the necessary fistula dilation rather than the stent design (in the case of the NAGI stent).

Spontaneous stent migration did not occur in any patient in our study. We had only 1 intraprocedural stent dislodgment during DEN due to impaction of a large fragment of necrosis. The stent was promptly replaced by a new one, without any clinical implication.

In our experience, the saddle shape of the LAMS is an effective design to achieve firm anchorage. We believe it also complements the single-step exchange-free technique which, given direct access to the stent, ensures a perfect fit between the saddle of the stent and the fistulous tract.

$\mathrm{H}_{2} \mathrm{O}_{2}$ irrigation was used in all patients. The rationale supporting its use is that $\mathrm{H}_{2} \mathrm{O}_{2}$ decomposes into water and oxygen when it combines with organic tissue, producing effervescence that mechanically cleans wounds and removes tissue debris via the released oxygen [33]. This helped debride necrotic material, which was then easily removed with accessories. As reported by Abdelhafez et al. [34] there are no AEs related to use of the low concentration of $\mathrm{H}_{2} \mathrm{O}_{2}$ in endoscopic treatment of WOPN. Siddiqui et al. [35] also reported a reduced need for mechanical debridement due to loosening of necrotic tissue over the course of hours to days.

In 4 patients, because of the massive extension and complexity of the collection, we decided to proceed with multiple drainage using 2 stents. The stents were deployed in 2 different sessions to achieve complete drainage of the collection. The second stent was placed if clinical conditions were not improving after first stent deployment. Subsequent necrosectomy was performed at each session through each of the different stents thus carrying out abundant and extensive $\mathrm{H}_{2} \mathrm{O}_{2}$ lavage.

We obtained complete resolution of WOPNs in more than 80 $\%$ of patients, and there was no recurrence after stent removal, which was uneventful in all patients.

In 2 patients, a pancreatic fistula was suspected at a control CT scan after LAMS removal due to presence of a pseudocyst. MRCP confirmed this suspicion and a pancreatic stent was then positioned in both cases, with resolution of the leak.

There were no AEs in our series. Although some groups have recently reported a worrisome $A E$ rate, our results differ from data in the literature. Bang et al. [36] reported in Gut their experience with electrocautery-enhanced LAMS in treatment of WOPN. They described a concerning $50 \%(6 / 12)$ rate of stentrelated $A E s$, in particular delayed bleeding (3/12). These results are different from what Bang et al. [37] reported for outcomes in 20 patients treated with electrocautery-enhanced LAMS compared retrospectively with 40 patients treated with double pigtail plastic stents. In that series, 13 of 20 patients treated with a LAMS had a WOPN. In this subgroup of WOPN patients, the stent-related AE rate was $15.4 \%$ (2/13). In both cases, minor AEs occurred without any delayed bleeding. The reason for such a large difference in terms of AE rates is unclear. It is possible that it is attributable to an undescribed change in technique.

As reported by Bang et al. [37], costs for treatment of WOPN with LAMS and plastic stents are nearly the same (US\$ 5,763.56 vs US $\$ 5,250.26$, respectively). Even if we did not include any cost analysis in our retrospective study, we believe that further prospective studies can demonstrate a reasonably reduced overall cost of treatment with LAMS for WOPN patients compared with treatment with plastic stents. This could be sustained by a significant impact on the total cost of the reported lower AE rates in patients treated with WOPN.

Our study has 2 limitations that require further comment. The first is that sample size was relatively small, though the number of total procedures was high (78).

The second limitation is that it is a retrospective assessment. 
The strengths of this study include the homogeneous cohort of patients, all with WOPN. Previous studies actually evaluated heterogeneous groups of patients with different types of pancreatic collection, including both WOPNs and pseudocysts. Furthermore, our study is the first in which the same type of stent was used, with the same positioning technique, i.e., 1-step, exchange-free, in all patients. Another strength of our study is the long-term follow up.

\section{Conclusions}

We conclude that endoscopic treatment of WOPN by positioning of LAMS and DEN is feasible, safe, and offers excellent results.

In particular we believe that the technical simplicity of the 1step, exchange-free positioning procedure for electrocautery enhanced LAMS plays a key role in terms of safety. To the best of our knowledge, our study registered the lowest complication rate $(0 \%)$ reported thus far in the literature, and a survival rate significantly higher compared with both surgical and percutaneous approaches.

\section{Competing interests}

None

References

[1] Dellinger EP, Forsmark CE, Layer P et al. Determinant-based classification of acute pancreatitis severity: an international multidisciplinary consultation. Ann Surg 2012; 256: 875-880

[2] Rau B, Bothe A, Beger HG. Surgical treatment of necrotizing pancreatitis by necrosectomy and closed lavage: changing patient characteristics and outcome in a 19-year, single-center series. Surgery 2005; 138: $28-39$

[3] Büchler MW, Gloor B, Müller CA et al. Acute necrotizing pancreatitis: treatment strategy according to the status of infection. Ann Surg 2000; 232: 619-626

[4] Rodriguez JR, Razo AO, Targarona J et al. Debridement and closed packing for sterile or infected necrotizing pancreatitis: insights into indications and outcomes in 167 patients. Ann Surg 2008; 247: 294 299

[5] Connor S, Alexakis N, Raraty MG et al. Early and late complications after pancreatic necrosectomy. Surgery 2005; 137: 499- 505

[6] Tsiotos GG, Luque-de León E, Sarr MG. Long-term outcome of necrotizing pancreatitis treated by necrosectomy. $\mathrm{Br}$ ] Surg 1998; 85 : $1650-1653$

[7] Howard TJ, Patel JB, Zyromski N et al. Declining morbidity and mortality rates in the surgical management of pancreatic necrosis. J Gastrointest Surg 2007; 11: $43-49$

[8] Baril NB, Ralls PW, Wren SM et al. Does an infected peripancreatic fluid collection or abscess mandate operation? Ann Surg 2000; 231: $361-367$

[9] Neff R. Pancreatic pseudocysts and fluid collections: percutaneous approaches. Surg Clin North Am 2001; 81: 399-403

[10] Seewald S, Ang TL, Kida M et al. EUS 2008 Working Group document: evaluation of EUS-guided drainage of pancreatic-fluid collections (with video). Gastrointest Endosc 2009; 69: S13-21
[11] Bakker OJ, van Santvoort HC, van Brunschot S et al. Endoscopic Transgastric vs Surgical Necrosectomy for Infected Necrotizing Pancreatitis. JAMA 2012; 307: 1053-1061

[12] Baron TH, Thaggard WG, Morgan DE et al. Endoscopic therapy for organized pancreatic necrosis. Gastroenterology 1996; 111: 755-764

[13] Yasuda I, Nakashima M, Iwai T et al. Japanese multicenter experienceof endoscopic necrosectomy for infected walled-off pancreatic necrosis: the JENIPaN study. Endoscopy 2013; 45: 627-634

[14] Gardner TB, Coelho-Prabhu N, Gordon SR et al. Direct endoscopic necrosectomy for the treatment of walled-off pancreatic necrosis: results from a multicenter U.S. series. Gastrointest Endosc 2011; 73: $718-726$

[15] Papachristou GI, Takahashi N, Chahal P et al. Peroral endoscopic drainage/debridement of walled-off pancreatic necrosis. Ann Surg 2007; 245: $943-951$

[16] Saxena P, Singh VK, Messallam A et al. Resolution of walled-off pancreatic necrosis by EUS-guided drainage when using a fully covered through-the-scope self-expandable metal stent in a single procedure (with video). Gastrointest Endosc 2014; 80: 319-324

[17] Talreja JP, Shami VM, Ku J et al. Transenteric drainage of pancreatic fluid collections with fully covered self-expanding metallic stents (with video). Gastrointest Endosc 2008; 68: 1199-1203

[18] Belle S, Collet P, Post S et al. Temporary cystogastrostomy with self expanding metallic stents for pancreatic necrosis. Endoscopy 2010; 42: $493-495$

[19] Fabbri C, Luigiano C, Cennamo V et al. Endoscopic ultrasound-guided transmural drainage of infected pancreatic fluid collections with placement of covered self-expanding metal stents: a case series. Endoscopy 2012; 44: 429-433

[20] Penn DE, Draganov PV, Wagh MS et al. Prospective evaluation of the use of fully covered self-expanding metal stents for EUS-guided transmural drainage of pancreatic pseudocysts. Gastrointest Endosc 2012; 76: 679-684

[21] Weilert F, Binmoeller KF, Shah JN et al. Endoscopic ultrasound-guided drainage of pancreatic fluid collections with indeterminate adherence using temporary covered metal stents. Endoscopy 2012; 44: 780 783

[22] Binmoeller KF, Shah J. A novel lumen-apposing stent for transluminal drainage of nonadherent extraintestinal fluid collections. Endoscopy 2011; 43: 337-342

[23] Itoi T, Binmoeller KF, Shah J et al. Clinical evaluation of a novel lumenapposing metal stent for endosonography-guided pancreatic pseudocyst and gallbladder drainage (with videos). Gastrointest Endosc 2012; 75: 870-876

[24] Shah RJ, Shah JN, Waxman I et al. Safety and Efficacy of Endoscopic Ultrasound- Guided Drainage of Pancreatic Fluid Collections With Lumen-Apposing Covered Self-Expanding Metal Stents. Clin Gastroenterol Hepatol 2014; 13: 747-752

[25] Rinninella E, Kunda R, Dollhopf M et al. EUS-guided drainage of pancreatic fluid collections using a novel lumen-apposing metal stent on an electrocautery-enhanced delivery system: a large retrospective study (with video). Gastrointest Endosc 2015; 82: 1039-1046

[26] Banks PA, Bollen TL, Dervenis C et al. Classification of acute pancreatitisd2012: revision of the Atlanta classification and definitions by international consensus. Gut 2013; 62: $102-111$

[27] Gornals JB, De la Serna-Higuera C, Sánchez-Yague A et al. Endosonography-guided drainage of pancreatic fluid collections with a novel lumen-apposing stent. Surg Endosc 2013; 27: 1428-1434

[28] Yamamoto $\mathrm{N}$, Isayama $\mathrm{H}$, Kawakami $\mathrm{H}$ et al. Preliminary report on a new, fully covered, metal stent designed for the treatment of pancreatic fluid collections. Gastrointest Endosc 2013; 77: 809-814

[29] Chandran S, Efthymiou M, Kaffes A et al. Management of pancreatic collections with a novel endoscopically placed fully covered self-ex- 
pandable metal stent: a national experience (with videos) Gastrointest Endosc 2015; 81: 127 - 135

[30] Walter D, Will U, Sanchez-Yague A et al. A novel lumen-apposing metal stent for endoscopic ultrasound-guided drainage of pancreatic fluid collections: a prospective cohort study. Endoscopy 2015; 47: $63-67$

[31] Mukai S, Itoi T, Baron TH et al. Endoscopic ultrasound-guided placement of plastic vs. biflanged metal stents for therapy of walled-off necrosis: a retrospective single-center series. Endoscopy 2015; 47: $47-55$

[32] Shah J, Shah JN, Waxman I et al. EUS-guided drainage of pancreatic pseudocysts (PP) utilizing a novel anchoring, covered self-expanding metal stent (Acsems): results from a prospective, multi-center study. Gastrointest Endosc 2013; 77: AB128
[33] Gruber RP, Vistnes L, Pardoe R. The effect of commonly used antiseptics on wound healing. Plast Reconstr Surg 1975; 55: $472-476$

[34] Abdelhafez M, Elnegouly M, Hasab Allah MS. Transluminal retroperitoneal endoscopic necrosectomy with the use of hydrogen peroxide and without external irrigation: a novel approach for the treatment of walled-off pancreatic necrosis. Surg Endosc 2013; 27: 3911 - 3920

[35] Siddiqui AA, Easler J, Strongin A et al. Hydrogen peroxide-assisted endoscopic necrosectomy for walled-off pancreatic necrosis: a dual center pilot experience. Dig Dis Sci 2014; 59: 687-690

[36] Bang JY, Hasan M, Navaneethan U et al. Lumen-apposing metal stents (LAMS) for pancreatic fluid collection (PFC) drainage: may not be business as usual. Gut 2016: DOI: 10.1136/gutjnl-2016-312812

[37] Bang JY, Hasan MK, Navaneethan U et al. Lumen apposing metal stents (LAMS) for drainage of pancreatic fluid collections: when and for whom? Dig Endosc 2017; 29: 83 - 90 